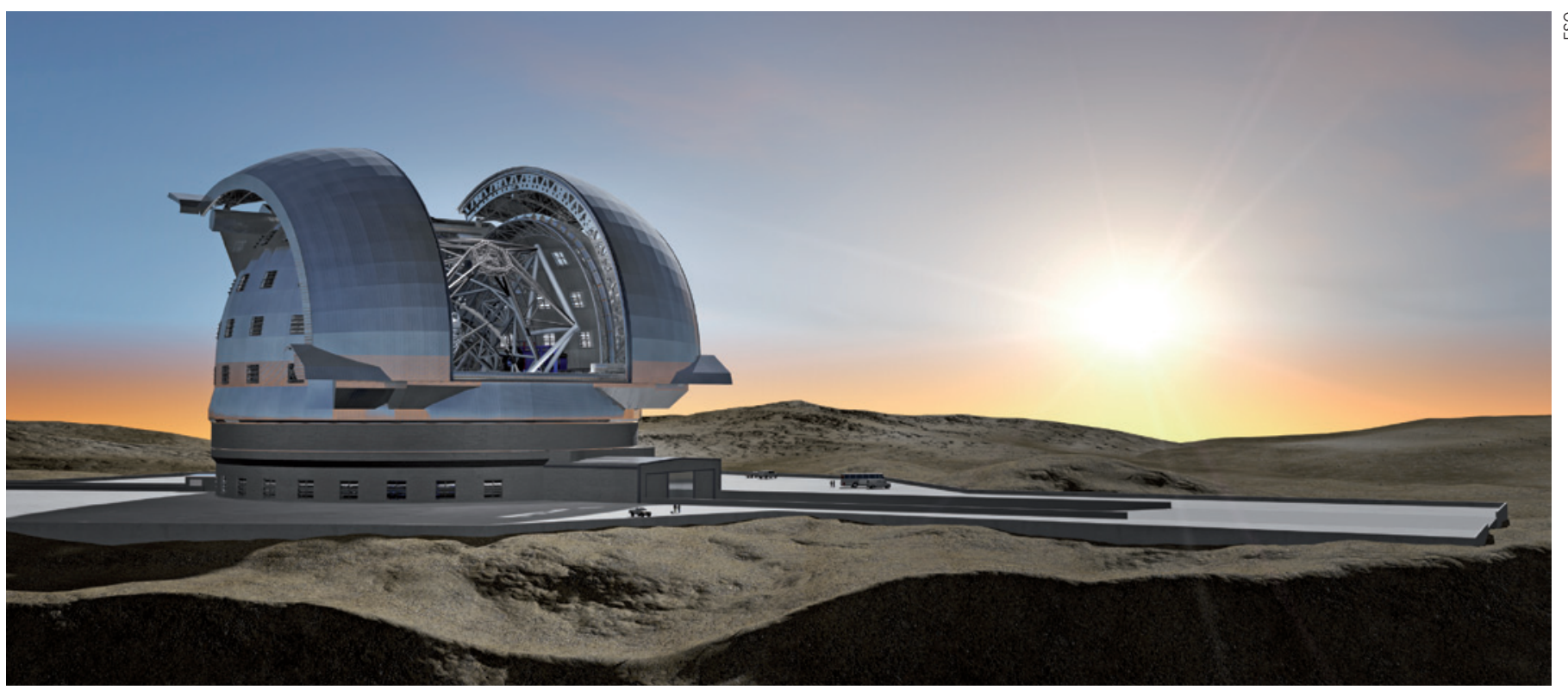

The European Extremely Large Telescope in Chile, as envisioned when completed around 2022, may be the first instrument to witness the birth of galaxies.

\title{
Megatelescopes look for support
}

\section{As a huge European observatory nears approval, a National Science Foundation funding competition between equivalent US projects remains in a holding pattern.}

\section{BY ERIC HAND}

A stronomers in the United States may have to do without government support in the race to create the world's biggest telescopes and gather photons from the Universe's first stars. Two projects - the Thirty Meter Telescope (TMT) and the Giant Magellan Telescope (GMT) - are vying for support from the US National Science Foundation (NSF), but the cash-strapped agency says that it may not be able to fund either before 2020 , by which time both projects had hoped to be finished (see Nature 469,451; 2011). That makes it even more likely that a comparable project, the European Extremely Large Telescope (E-ELT), will be ready years ahead of its US-led counterparts.

Jim Ulvestad, director of the NSF's astronomy division, is charged with holding a competition to choose which project the agency should support - a move emphatically recommended by the 2010 decadal survey in astronomy, an assessment of priorities for US agency planners. "But the decadal survey also assumed we'd have a lot more money than we do," Ulvestad says.

The problem, says Ulvestad, is a long queue of major new facilities that the NSF is already committed to funding between now and the end of the decade (see 'Join the queue'). Even the cancellation of the Deep Underground Science and Engineering Laboratory in December
2010 (see Nature http://dx.doi.org/10.1038/ news. $2010.683 ; 2010)$ is not enough to overcome a poor allocation for such projects from Congress earlier this year.

Ulvestad says the agency may still launch a competition in December or January although the winner would not be guaranteed any money. The competition could coincide with approval of the 39-metre E-ELT, planned for Cerro Armazones in Chile. At a council meeting in December, member states of the European Southern Observatory (ESO), based in Garching, Germany, plan to authorize limited construction on the $€ 1$.1-billion (US\$1.6-billion) telescope - a move that could lure frustrated international partners away from the two US-led efforts.

The US teams are at odds in their views on an
NSF competition. The consortium behind the \$1-billion TMT, planned for the top of Mauna Kea in Hawaii, thinks it has a good chance of besting its rival; it has lobbied for years for the NSF to pick a winner. "Let's get on with it," says Richard Ellis, a member of the TMT board and an astronomer at the California Institute of Technology in Pasadena. "Let's at least make plans, even if the money comes later."

Ellis says that NSF backing would help the TMT to nail down other partner nations, especially China, which wants to see US government action before it commits completely. $\mathrm{He}$ adds that even if the NSF lacks money for construction, it could become a partner by paying operating expenses later on. And holding a competition might compel the agency to find money in the future. "The NSF has to rise to

\footnotetext{
JOIN THE QUEUE

Commitments to a succession of projects (left) with large capital costs (right) over the next 10 years may keep the US National Science Foundation from contributing to a large US-led telescope until 2020. PROJECT ALMA $\ll$. Started $2002 \cdots \cdots \cdots \cdot \cdots$

Advanced LIGO $<.$. Started 2008..........

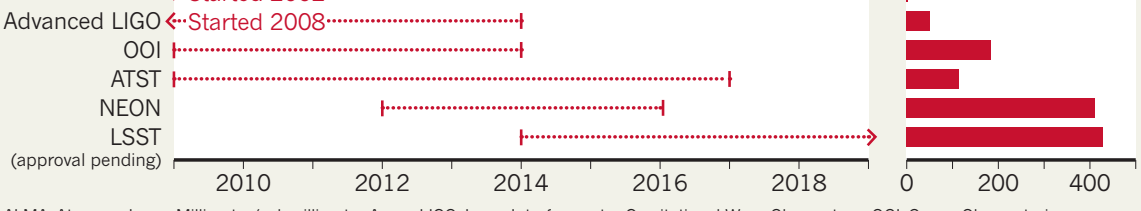

ALMA, Atacama Large Millimeter/submillimeter Array; LIGO, Laser Interferometer Gravitational Wave Observatory; OOI, Ocean Observatories Initiative; ATST, Advanced Technology Solar Telescope; NEON, National Ecological Observatory Network; LSST, Large Synoptic Survey Telescope.
} 
the challenge of lobbying for facilities," says Ellis. "It has been too passive."

The GMT prefers the passive approach. The 25-metre telescope, planned for Las Campanas, Chile, is the smallest of the projects, and will be about $\$ 300$ million cheaper than the TMT. The involvement of wealthy institutions such as Harvard University in Cambridge, Massachusetts, is assured, so the consortium might be able to raise its capital without the NSF. It already has something to show for its efforts: astronomers cast the first of the GMT's seven mirrors in 2005, and since then have been configuring it to a unique off-axis reflecting surface. Wendy Freedman, chairwoman of the GMT board and director of the Carnegie Observatories in Pasadena, says that the GMT still wants to partner with the NSF, but would prefer to hold off on a competition.

"Making decisions without money strikes us as an unusual thing to do," she says. "The logical thing to do would be to wait." She doesn't agree that NSF backing will help to attract partners; the GMT already has financial commitments from institutions in Australia and South Korea. Ultimately, says Freedman, the GMT may elect not to compete.

The ESO has a steady stream of funding, but even so, the E-ELT's price tag could prove problematic. Current treaty-enforced annual fees from the 14 member states will account for only about one-third of the cost. Brazil became the source for another third when it agreed in December 2010 to join the ESO. And on 13 October, Chile formally donated the site on which the E-ELT will be built.

But the observatory still has to find the remaining third of the cost, says Tim de Zeeuw, director-general of the ESO. One way would be to recruit yet more members. De Zeeuw notes that a number of countries are "asking questions" about membership: Russia, Poland, Canada (currently a TMT member) and Australia (committed to the GMT). But in the meantime, de Zeeuw is trying to muster approval from the member states for an annual $2 \%$ fee increase, along with a one-time special contribution proportional to the state's income.

Three nations - Sweden, Finland and the Czech Republic - have agreed to unlock the money, he says. But the remaining 11 are not likely to rally approval by December; nor is the Brazilian government expected to have ratified its membership treaty by then.

Approval of the full E-ELT budget won't come before a meeting in March 2012. Instead, the council is planning to approve early contracts and crucial infrastructure, such as a road to the E-ELT's mountain location. But even this limited construction authority will give the project momentum. "They could have said, 'We'll decide next year,'” says Roberto Gilmozzi, principal investigator of the project.

The US competition, once begun, would probably be decided in less than a year. Ulvestad says that the NSF would want to evaluate the risk of the projects, and would ask how, for instance, the telescope consortia might provide publicly accessible archives or user support for astronomers not associated with consortium institutions.

But Ulvestad also realizes that, with so long until the money will materialize, the telescopes might be completed without the NSF's help. Much of the US academic astronomy community relies on NSF support to get access to telescope time, which might not be possible with the giant telescopes unless the NSF has contributed to them. But, he says, with a total of seven US universities signed on to the TMT or GMT, there is already "a lot of US community in their partnerships".

\section{CORRECTION}

The News story 'Malaria vaccine results face scrutiny' (Nature 478, 439-440; 2011) wrongly described Plasmodium falciparum as multicellular. The parasite is unicellular. 